\title{
A Microdosimetry Analysis of Reversible Electroporation In Scattered, Overlapping, And Cancerous Cervical Cells
}

\author{
Mayank Kumar ( $\nabla$ rss2018501@iiita.ac.in ) \\ Indian Institute of Information Technology Allahabad \\ Ashutosh Mishra \\ Indian Institute of Information Technology Allahabad
}

\section{Research Article}

Keywords: numerical, electroporation, microdosimetry, experimental

Posted Date: November 17th, 2021

DOl: https://doi.org/10.21203/rs.3.rs-898413/v1

License: (c) (i) This work is licensed under a Creative Commons Attribution 4.0 International License.

Read Full License 


\title{
A microdosimetry analysis of reversible electroporation in scattered, overlapping, and cancerous cervical cells
}

\author{
Mayank Kumar ${ }^{1, *}$ and Ashutosh Mishra ${ }^{2}$ \\ ${ }^{1}$ Indian Institute of Information Technology Allahabad, Department of Applied Sciences (Biomedical Engineering), \\ India \\ *rss2018501@iiita.ac.in \\ +am@iiita.ac.in
}

\section{ABSTRACT}

In this paper,a numerical method for studying reversible electroporation on normal and cancerous cervical cells is introduced. This microdosimetry analysis has been done by a unique approach for extracting contours of free and overlapping cervical cells in the cluster from the External Depth field images ${ }^{19}$. The algorithm used for extracting the contours is a joint optimization of multiple level set function along with the Gaussian mixture model and Maximally Stable Extremal Regions. This contour is then imported a multiphysics domain solver, where variable frequency pulsed electric field is applied. The Trans-Membrane Voltage (TMV) developed across the cell membrane is then calculated using the Maxwell equation coupled with a statistical approach employing the asymptotic Smoluchowski equation, which calculates the generated temporal pore density. The numerical model was validated by successful replication of existing experimental approach that employed low-frequency uni-polar pulses on the overlapping cells to obtain reversible electroporation. Using several overlapping clumps of cervical cells, simulations are performed to match the experimental data. For high-frequency calculation, a combination of normal and cancerous cells is introduced to the computational domain. The cells are assumed to be dispersive and the Debye dispersion equation is a second-order partial derivative equation used for further calculations. The difference in time duration for reaching the threshold value of electroporation is seen between the normal and cancerous cervical cells due to their size and conductivity change. The drug and dye uptake modulation during the high-frequency electric field electroporation is advocated by a mathematical model.

\section{Introduction}

Electroporation is an electrical process in which the biological cells and tissues are exposed to (usually - pulsed) electric field, leading to the formation of pores across the cell membrane, which can be used as a transport channel to/from the cells. There exist - two forms of electropration - reversible and irreversible. IRreversible Electroporation (IRE) is when the pores formed are permanent and generally used for cellular ablation or apoptosis. Reversible Electroporation (RE) is where the exposures to electrical pulses cause pore formation but these pores reseal after some time. This method is used for drug delivery and DNA transfer ${ }^{1,2}$. Microdosimetry is used for studying the interaction of electrical energy with biological matter. It has been frequently used for electroporation study since the real process of electroporation is still being investigated, as evidenced by several numerical studies. The in vitro electroporation study uses an experimental-driven approach and model-driven approaches. The model-driven approach can benefit as the prolonged process of trials and test leading to wastage of cells in the experiments could be saved with the availability of a proper numerical model ${ }^{3,4}$. Most of the numerical study has been done using regularly and irregularly shaped cells giving spatial distribution of the membrane potential ${ }^{5-9}$. The morphology of single and packed cells has been extracted from neuroblastoma cells for microdosimetry study, which exhibited a decrease in TMV for packed cells when compared to the isolated cells ${ }^{10}$. Confocal microscopy has been used to reconstruct the $3 \mathrm{D}$ cell ${ }^{11}$ and $\mathrm{RE}$ has been achieved both numerically and experimentally. The $3 \mathrm{D}$ construction is also used to create the topology of the endoplasmic reticulum in the work presented ${ }^{4}$ to develop an electroporation model.

The most frequent cancer found among women in the world is cervical cancer. The liquid-based in vitro cytology test or Pap smear test is the most screening test, employed for the identification of cancerous cells ${ }^{12,13}$. The Cervical Intraepithelial Neoplasia (CIN) is the abnormal growth of the cells that are seen in the cervical tissue, which undergoes three phases of change known as CIN1, CIN2, and CIN $3^{14,15}$. Most of the work done is based on irreversible electroporation, where ablation of the cell is done by the application of pulsed electric field with the time period $25 \mu s-100 \mu s$ and pulse amplitude from $500 \mathrm{~V} / \mathrm{cm}$ to $4.5 \mathrm{KV} / \mathrm{cm}$ with repeated pulses applied at a frequency of $1 \mathrm{~Hz}^{16}$. These experiments were carried out in vitro with a mono-layer cell line. Twenty five Gaussian 
pulses were applied for $70 \mathrm{~ns}$ and $25 \mathrm{KV} / \mathrm{cm}$ electric field strength to achieve thermal ablation of cervical cancer cells $^{17}$. A realistic numerical model of single $2 \mathrm{D}$ cervical cells has been proposed in which nanosecond electrical pulses produced by a Marx generator were applied. Numerical simulation reveal that the cells achieved the threshold TMV and pore density ${ }^{18}$.

First an experiment-driven approach is taken, in which RE on overlapping and isolated cells is induced by utilizing the electric field and duration from the experimental data. The pulse strength, duration, and pulse count applied are evaluated on the basis of high cell viability and permeability. The cervical cells extra depth field images were prepared in Australian Centre for Visual Technologies, University of Adelaide. The use of simple image processing techniques and availability of cervical cell extra depth field (EDF) images in the database ${ }^{19}$ is used to extract the contour of the cell. The algorithm is implemented to get the contour of various overlapping cells, isolated cells and a combination of both to yield a heterogeneous cluster of cancerous and normal cells. This topology is imported to the multiphysics area for further simulations. The contour is vectorized to carry on the multiphysics analysis. The results of the model and experimental data show high levels of agreement with each other regarding pore density and TMV. The threshold electric field for high-frequency uni-polar pulses is used in the diffusion analysis for exploring the electrically mediated uptake of dyes or drugs into the cervical cell. A faster development of the required TMV and pore density is observed in the cancerous cervical cells. The proposed model shows considerable promise for the development of model driven microdosimetry. The modulated uptake of dyes into cells could be further used for observing change in morphology of the cells while the uptake of drugs could be used for therapeutic purposes.

\section{Results}

The cervical cells are first exposed to uni-polar rectangular pulses of low-frequency pulse of time duration $100 \mu s$ and electric field strength of $1 \mathrm{KV} / \mathrm{cm}$. Different Cellular clusters and isolated cell configurations are subjected to the electric stress, as shown in Figures 1-3. The computational domain of $500 \mu \mathrm{m} \times 500 \mu \mathrm{m}$ is used, and copper electrodes $(500 \mu \mathrm{m} \times 10 \mu \mathrm{m})$ of resistance $0.0038 \Omega$ are attached to the top and bottom of the rectangular domain. The arrow indicates the orientation of the applied electric field. The points noted in each figure are selected from the contour for analysis to highlight the effect of the applied electric field on overlapping cells and free cells (as applicable). The temperature analysis is done to verify that no thermal ablation is seen at the applied pulse indicating RE. The contours are extracted using the image processing algorithm, detailed in the methodology section. The TMV and pore density are plotted at these points to evaluate whether the membrane(s) have reached the threshold pore density of $10^{14} \mathrm{~m}^{-2}$ and TMV 1-1.5 V. These results are provided to show good validation of our numerical model with existing experimental results ${ }^{16}$. The temperature rise is not enough to ablate the cells demonstrating safe thermal operating point. In Figures 1-3, plot (a) shows the cluster image with the selected points P1-P5,P4,P7. Plots (b) and (c) show the TMV (V) and the pore density respectively (computed at $50 \mu s$ ). The temperature map is plotted at $100 \mu \mathrm{s}$ in plot (c) showing a profile that spans $310 \mathrm{~K}$ to $318 \mathrm{~K}$ - which is not sufficient for cells to undergo ablation. Plots (e) and (f) show the temporal development of the TMV and the pore density.

Having successfully validated our proposed model, a high-frequency pulse is applied to a mixture of cancerous and normal cervical cells. Figure 4. (a) shows a mixture of superficial normal and cancerous cervical cells. The stages of cancer progression in the superficial cervical cells - CIN1, CIN2, and CIN3 are shown in the figure. These cells are introduced in a similar computational domain of $500 \mu \mathrm{m} \times 500 \mu \mathrm{m}$. The electric field of $10 \mathrm{kV} / \mathrm{cm}$ for $40 \mathrm{~ns}$ is applied on the copper electrodes (described earlier). Figure 4. (b-c) show the TMV and pore density map of the membrane, captured at 20 ns. Figure 5-a shows the TMV plot of four cells from P1-P4 where P1 is the point at the superficial cell, P2 is the point at first stage cancer cell stage CIN1, P3 is the point at second cancer cell stage CIN2 and $\mathrm{P} 4$ is the point at third stage cancer cell CIN3. We note that the rate of TMV evolution is faster in CIN3 (CIN3 $>$ CIN2 $>$ CIN1 $>$ normal cells). Figure 5-b shows that the pore radius evolution is faster in CIN3 and a similar order is observed. The pore density evolution shown in figure 5 -c exhibits a similar pattern. The rise in conductivity of the cell membrane is shown in Figure 5-d. We also explored the dye diffusion under electric stress through this high-frequency pulse application. The extracellular fluid was setup with a dye concentration $0.01 \mathrm{~mole} / \mathrm{m}^{3}$. The uptake of the dye in the cells at points P1-P4 is shown in Figure 5-e, which highlights that CIN3 cells, due to ease of electroporation and with larger pore density, exhibit faster uptake compared to other cells. Figure 5-f shows no considerable rise in the temperature of cells, but a relatively larger rise is observed in CIN3 cells. The polarization plot in Figure 5-g shows that the CIN3 cells undergo higher polarization and at a faster rate. 


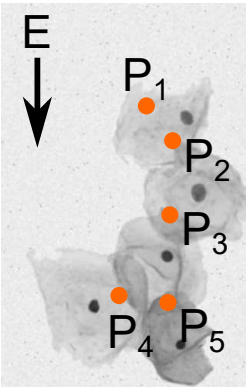

(a)

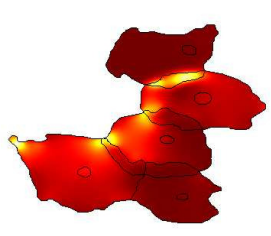

(d)

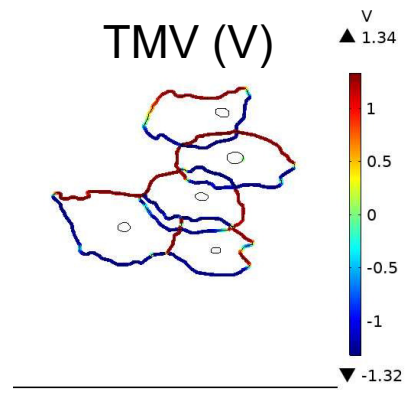

(b)

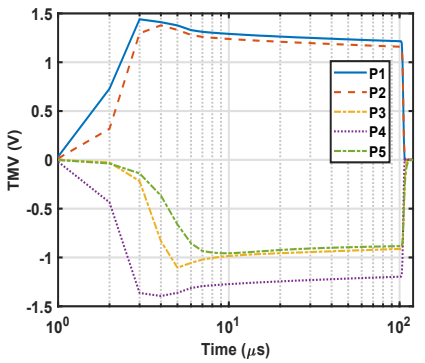

(e)

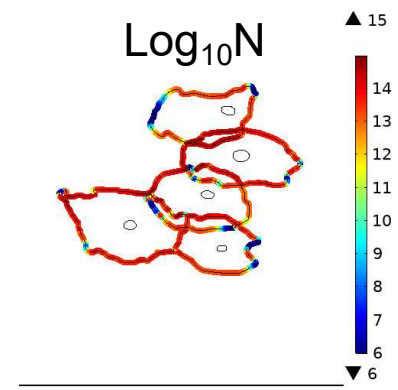

(c)

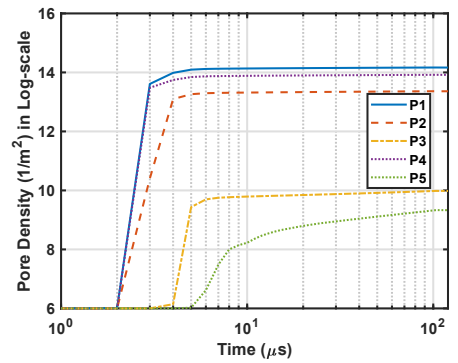

(f)

Figure 1. (a) EDF image of the cervical cells (b) TMV contour map at $50 \mu s$ (c) Pore Density plot in Log scale at $50 \mu s$ (d) Temperature (K) map at 100 $\mu$ s (e) Temporal devlopement of TMV at P1-P5 (f) Temporal development of pore density in Log-scale at P1-P5.

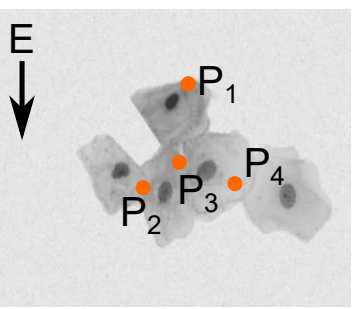

(a)

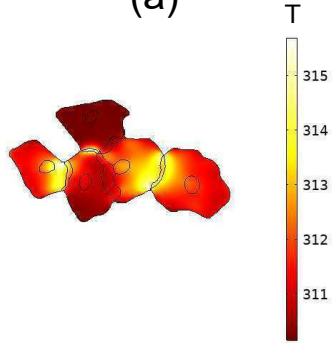

(d)

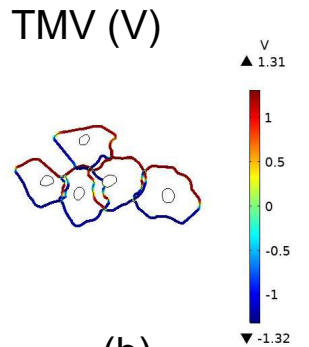

(b)

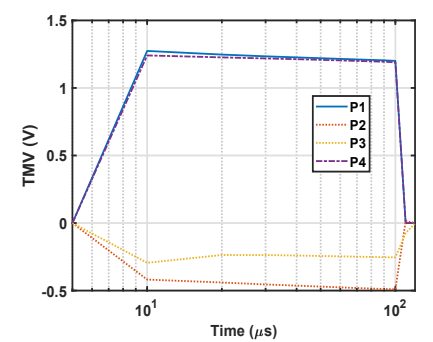

(e)

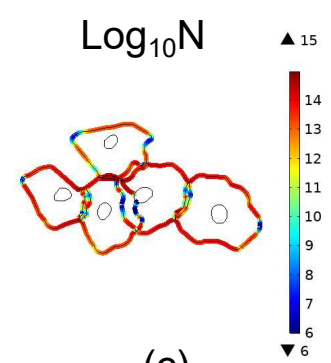

(c)

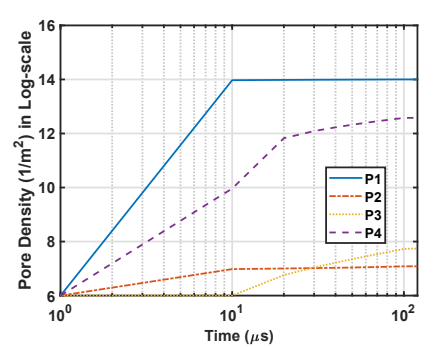

(f)

Figure 2. (a) EDF image of the cervical cells (b) TMV contour map at $50 \mu \mathrm{s}$ (c) Pore Density plot in Log scale at $50 \mu s$ (d) Temperature (K) map at $100 \mu s$ (e) Temporal devlopement of TMV at P1-P4 (f) Temporal development of pore density in Log-scale at P1-P4. 


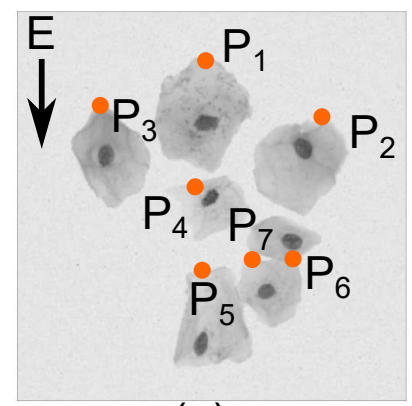

(a)

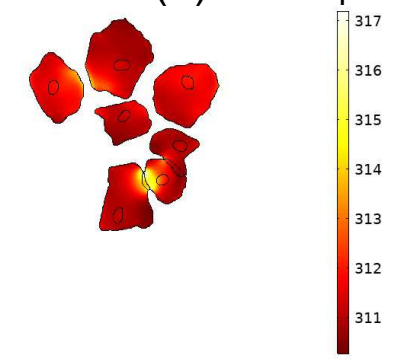

(d)

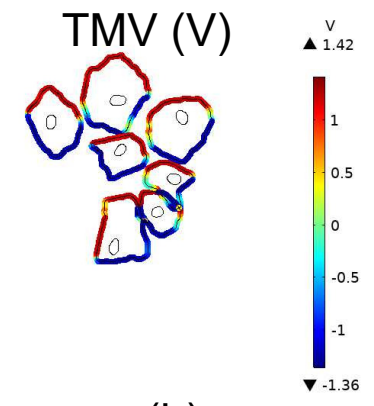

(b)

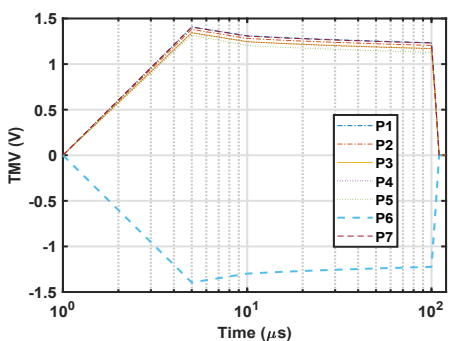

(e)

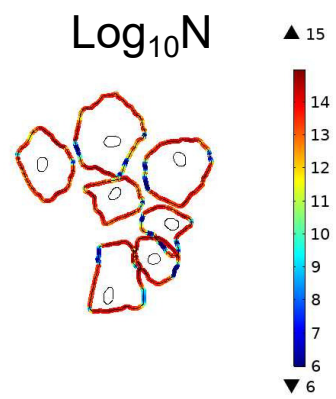

(c)

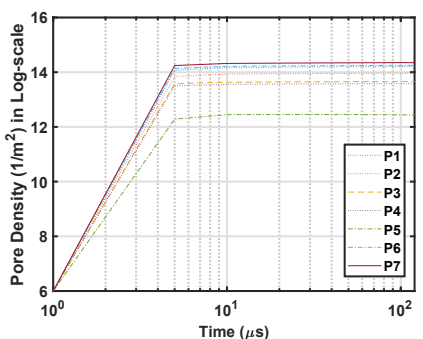

(f)

Figure 3. (a) EDF image of the cervical cells (b) TMV contour map at $50 \mu s$ (c) Pore Density plot in Log scale at $50 \mu s$ (d) Temperature (K) map at $100 \mu s$ (e) Temporal devlopement of TMV at P1-P7 (f) Temporal development of pore density in Log-scale at P1-P7.

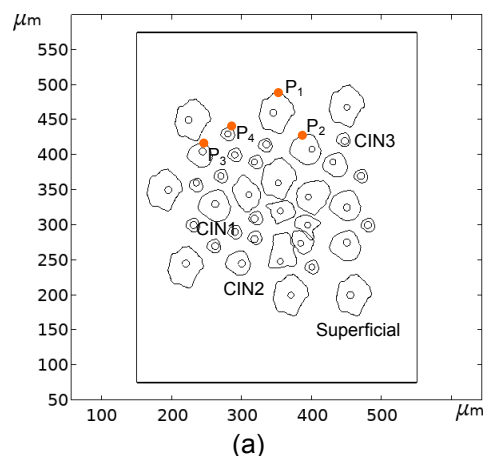

(a)

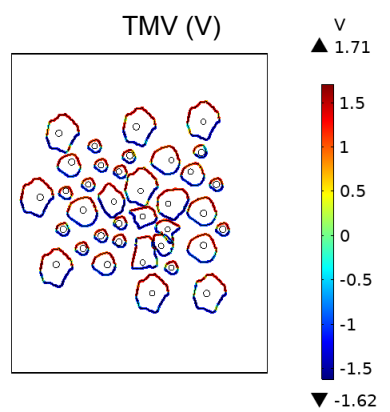

(b)

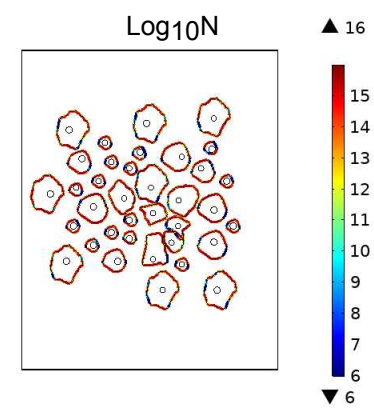

(c)

Figure 4. (a) Cervical normal and cancerous cells computational domain (b) TMV contour map at 20 ns (c) Pore $\operatorname{density}\left(1 / \mathrm{m}^{2}\right)$ contour map at $20 \mathrm{~ns}$. 


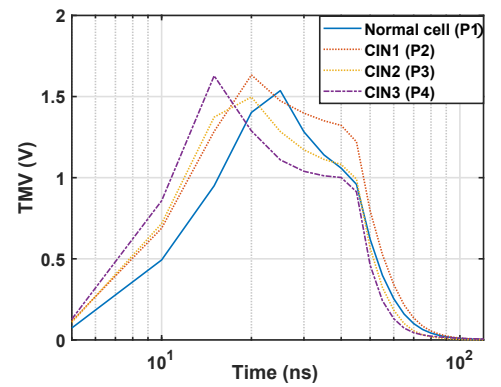

(a)

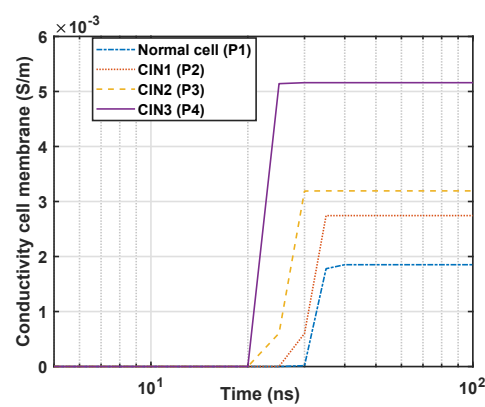

(d)

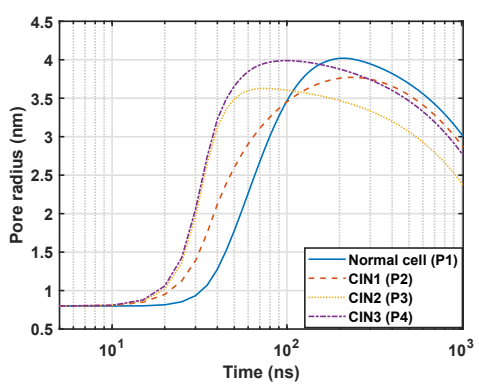

(b)

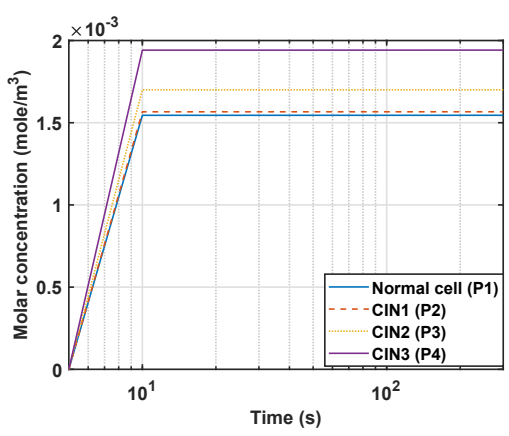

(e)

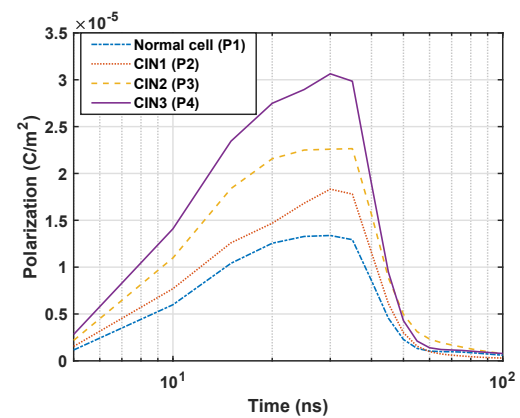

(g)

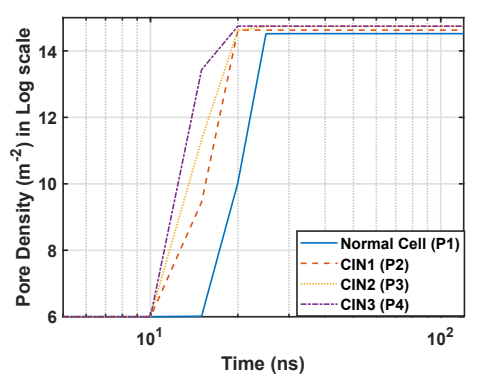

(c)

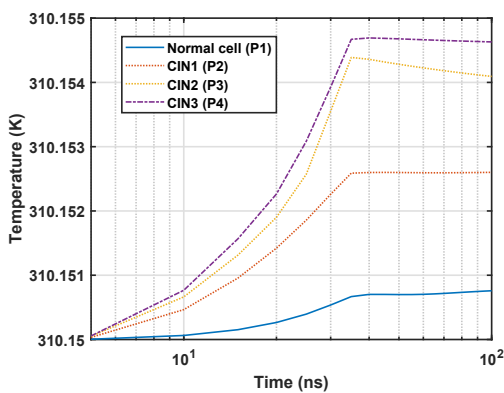

(f)

Figure 5. (a) TMV (V) temporal evolution at $\mathrm{P} 1$ to $\mathrm{P} 4$ (b) Pore radius(nm) temporal evolution at $\mathrm{P} 1$ to $\mathrm{P} 4$ (c) Pore density temporal evolution at P1 to P4 (d) Temporal rise in conductivity of cell membrane from P1-P4 (e) Molar $\left(\mathrm{mole} / \mathrm{m}^{3}\right)$ intake of dye into the cells (f) Temperature rise at points P1-P4 with time (g) Temporal plot of polarization at point P1-P4.

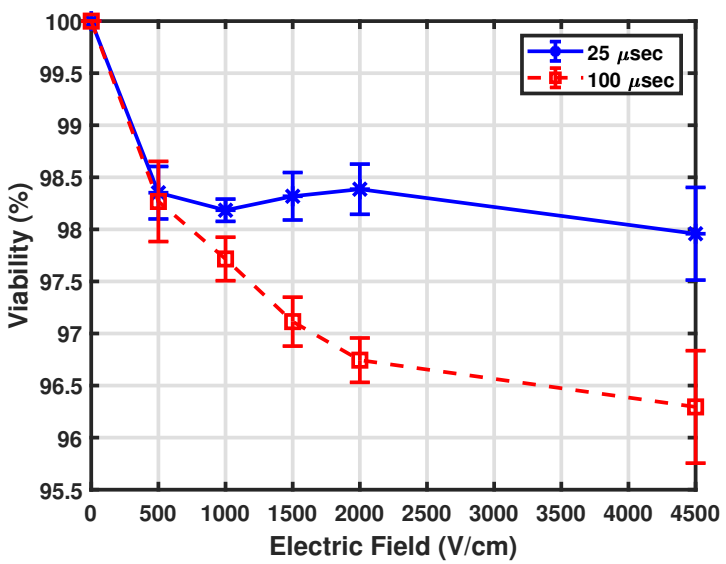

Figure 6. Experimental data plot of viability (\%) and Electric field $(\mathrm{V} / \mathrm{cm})$ uni-polar rectangular pulse. 


\section{Discussion}

Experimental data ${ }^{16}$ has been used to evaluate the pulse strength and duration to establish concurrence with our proposed numerical model. As shown in Fig-6, the required strength of the electric field is around the $1 \mathrm{KV} / \mathrm{cm}$ where the viability is $97.625 \%$ for $100 \mu s$ pulse. This is the point of threshold where the cervical cells, when exposed to the electric field, have higher vitality and permeability ${ }^{16}$. The plots in Figures 1-3 have all the results of the overlapping and isolated cells which have been reversibly electroporated. These plots also include the thermal profile, which shows that temperature rise observed in the cells is not enough to cause ablation. The overlapping zones exhibit marginally lower level of electroporation as cells behave as a single unit or more like a single large cell. A pulse strength of $10 \mathrm{KV} / \mathrm{cm}$ was also applied to the model for $40 \mathrm{~ns}$ in order to reversibly electroporate the cell to modulate the dye uptake by the cells. This is driven by the assumption that the dispersive model (using the Debye second order expression) would have a lower threshold of the electric field as polarization is observed at these high-frequency levels - which is a reflection of dispersion in an electrical medium. The cellular clusters simulated here included normal and cancerous cervical cells. The cancerous cells exhibit a faster development of TMV and threshold pore density owing to an increase in conductivity and decrease in size with increasing levels of neoplasia. The intake of drugs or dyes in cancerous cells is predicted to be higher than the normal cells due to higher pore density, as shown in Figure 5-e.

\section{Methods}

\section{Cervical cells contour extraction}

The initial stage is to extract only the cell clusters without the background artifacts so that all the analysis stays on cervical cells from Figure 7 -a. This process starts with application of quick shift algorithm ${ }^{20}$ which is a variant of mean shift to find local maxima density. The result is a creation of super-pixel grey scale map. An edge detector applied to this super-pixel map removes an residual background artifacts. Subsequently, this clean map is fed to an unsupervised learning algorithm for separating the cell clump from the total map dimensions. A brief over view of this process is as follows: A connected component analysis is done on the edge map so that a convex hull can be built inside these components. The components lying in the convex hull belong to the cells and rest background. The technique uses development of Gaussian Mixture Models (GMMs) through maximum likelihood estimation where, the feature extracted from the pixel consists of its greyscale value. The ratio of likelihood between the two classes of each pixel is used for classification. The re-estimation process of likelihood map is repeated until a stable GMM is achieved. This GMM includes some noise from those areas of image which are not eliminated by the edge detector. This is finally removed by a post processing application of a k-means clustering algorithm. The Figure 7-b shows the derived outer boundary of the cluster through this process.

The nucleus detection is an important part of this algorithm as it is used to create a shape prior and subsequent level set method optimization after initial segmentation. The nucleus can be easily differentiated due to its low gray value, almost circular geometry and homogeneous texture. The assumption made here is that nuclei do not overlap each other. The Maximum Stable Extremal Region (MSER) ${ }^{21}$ is applied on the cell clumps obtained from the process mentioned above for nucleus detection. The Figure 7-c shows the isolated nuclear regions within the cellular clump after implementing the MSER algorithm on the image 7-a.

A shape prior is built from the segmented images (Figure 7-b \& 7-c), which is further used for level set optimization. In order to build shape prior, each point on the clump boundary is associated to its nearest nucleus so that the cell ownership could be claimed. To ensure smoothness of the boundary, segmented regions are interpolated using the Delaunay triangulation (implemented by the function TriScatteredInterp)in MATLAB. In the overlapping region this function draws the cell boundaries of each cell by following the extreme clump points. The geometric center (centroid) of each cell after interpolation of the boundaries is also calculated. The boundary is then used for level set optimization. For each cell in the clump, following constraints were used:

$$
h_{i}(x)=\left\{\begin{array}{lr}
1, & \text { if } \mathrm{x} \text { outside clump C } \\
2+\frac{-2}{(1+\exp (-\beta t(x))}, & \text { if } \mathrm{x} \text { inside cell } \mathrm{i} \\
0, & \text { otherwise },
\end{array}\right\}
$$

where $\mathrm{C}$ represents the set of clumps $\mathrm{C}=\left\{h_{i}\right\}_{i=1}^{|C|}$ with $|C|$ denoted by $h_{i}$. The function $t(x)$ denotes distance between the point $x$ on the 2D image domain and the geometric center of the initial segmentation, $\beta$ represents a 
heuristic free parameter. The shape prior constant is then defined as

$$
h_{C}(x)=\max \left(h_{i}(x)\right)
$$

where $h_{i} \in C$

A joint level set optimization is used in this work in which the shape prior is used as shown in Figure 7-d. $\phi(x, y, t): \Omega \rightarrow \mathbb{R}$ denotes a 2D time dependent Level Set Function (LSF) with N cells that have been detected. LSF is denoted by $\left\{\phi_{i}\right\}_{i=1}^{N}$ and the energy function to be minimized is given by equation

$$
\epsilon\left(\left\{\phi_{i}\right\}_{i=1}^{N}\right)=\Sigma_{i=1}^{N}\left(\epsilon_{u}\left(\phi_{i}\right)+\Sigma_{i=1}^{N} \Sigma_{j \in N_{(i)}}\left(\epsilon_{b}\left(\phi_{i}, \phi_{j}\right)\right)\right.
$$

where $\epsilon_{u}$ is the unary energy function for each LSF,$\epsilon_{b}$ represents the binary function over LSF and $N(i)$ is the pixel index at the point of intersection of the level set function $\phi_{j}$ with the zero level set function of $\phi_{i}$. The unary function $\epsilon_{u}$ is defined as:

$$
\epsilon_{u}\left(\phi_{i}\right)=\mu R\left(\phi_{i}\right)+\kappa D\left(\phi_{i}\right)
$$

where $\mu>0 \& \kappa \in \mathbb{R}$ represents the iteration factor. The first regularization term :

$$
R\left(\phi_{i}\right)=\int_{\Omega} p(|\nabla \phi|) d x
$$

where $p:[0, \infty] \rightarrow \mathbb{R}$ and $|\nabla \phi|=1$ is the regularized term that maintains the signed distance property only in the vicinity of zero level set and $|\nabla \phi|=0$ for far away points from the zero level set.

$$
D\left(\phi_{i}\right)=\int_{\Omega} h_{C}(x) g \delta\left(\phi_{i}\right)(|\nabla \phi|) d x
$$

where $h_{C}(x)$ is the shape prior constant as stated above which defines the pixel features stated in equation 1 for a clump and here $g=\frac{1}{1+\left|\nabla G_{\sigma} * I\right|}$ where $I$ represents the image edge information $\left(G_{\sigma}\right.$ represents Gaussian kernel at that standard deviation). $D\left(\phi_{i}\right)$ is derived from the Geodesic active contour length term provided. The regularization term $R\left(\phi_{i}\right)$, guarantees $|\nabla \phi|=1$ but to maintain the compatibility with the literature this term is added ${ }^{22}$. The $D\left(\phi_{i}\right)$ is minimized until shape of the cell is same as the shape prior and zero level set is located in the image domain with largest gradient.

$$
\epsilon_{b}\left(\phi_{i}, \phi_{j}\right)=\chi \int_{\Omega} h_{C}(x) g H\left(-\phi_{i}\right) H\left(-\phi_{j}\right) d x
$$

where $\chi>0, H()$ is the Heaviside function. The regions where $h_{C}(x)$ value is low, the function value is minimized which represent areas close to cell borders and far away from the nuclei and contains large gradient values of image. For finding minimum energy of the function $\epsilon\left(\left\{\phi_{i}\right\}_{i=1}^{N}\right)$ a gradient flow equation of the the each LSF $\phi(x, y, t)$ is given as:

$$
\frac{\partial \phi_{i}}{\partial t}=-\frac{\partial \epsilon\left(\left\{\phi_{i}\right\}_{i=1}^{N}\right)}{\partial \phi_{i}}
$$

where $\frac{\partial \epsilon\left(\left\{\phi_{i}\right\}_{i=1}^{N}\right)}{\partial \phi_{i}}$ represents the Gateaux derivative of the function $\epsilon\left(\left\{\phi_{i}\right\}_{i=1}^{N}\right)$ which follows the steepest direction of the function \& solved using usual finite difference schemes. Based on equation $1^{23}$

$$
\frac{\partial \epsilon\left\{\phi_{i}\right\}_{i=1}^{N}}{\partial \phi_{i}}=\frac{\partial \epsilon_{u}\left(\phi_{i}\right)}{\partial t}+\sum_{j \in N(i)} \frac{\partial \epsilon_{b}\left(\phi_{i}, \phi_{j}\right)}{\partial \phi_{i}}
$$

These equations are implemented in the Algorithm 1 and the contour is obtained - as shown in Figures 7 (a-e). After obtaining the required contour of cervical cells they are reshaped to the size provided in Table $1^{14,15}$. 


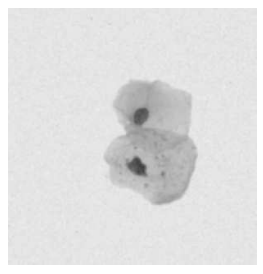

(a)

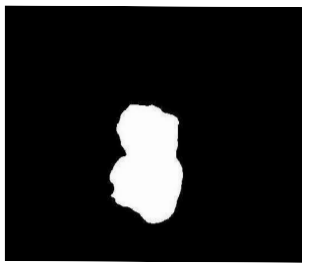

(b)

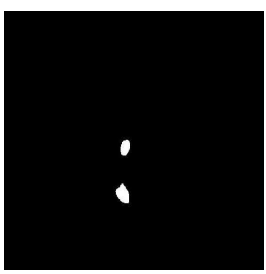

(c)

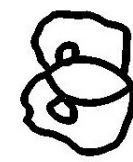

(d)

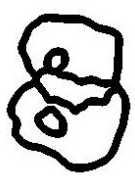

(e)

Figure 7. (a) Cytology cervical cell image (b) Clump obtained after GMM (c) Nucleus mask obtained by MSER algorithm (d) Initial shape prior formed (e) Original Contour of the cell after LSF evolution.

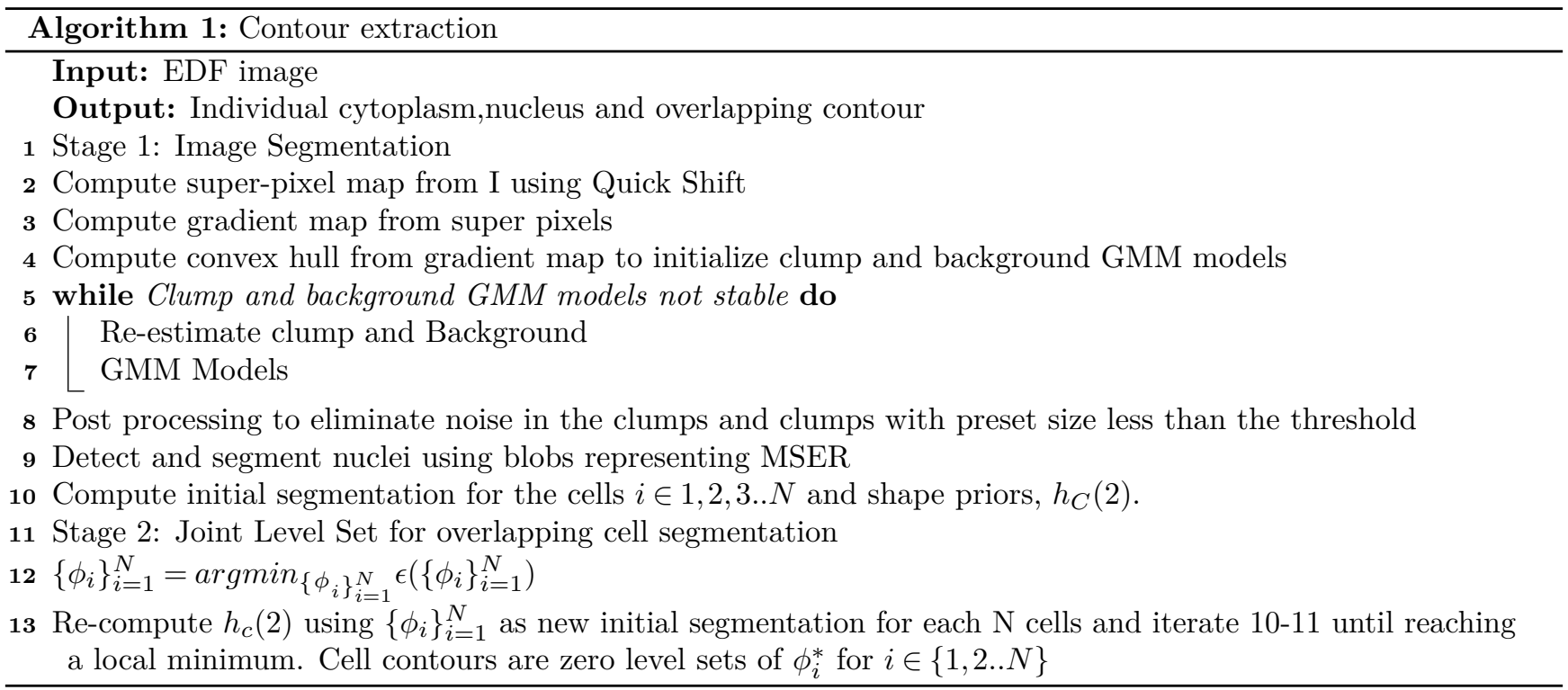


Table 1. Cervical cell dimension table

\begin{tabular}{|c|c|c|c|c|}
\hline Cell types & \multicolumn{2}{|c|}{ Cell } & \multicolumn{2}{c|}{ Nucleus } \\
\hline & $\mathrm{X}$ & $\mathrm{Y}$ & $\mathrm{X}$ & $\mathrm{Y}$ \\
\hline Superficial & $66 \pm 9.2 \mu \mathrm{m}$ & $66 \pm 9.2 \mu \mathrm{m}$ & $8.4 \pm 1.1 \mu \mathrm{m}$ & $8.4 \pm 1.1 \mu \mathrm{m}$ \\
\hline Intermediate & $52 \pm 10 \mu \mathrm{m}$ & $52 \pm 10 \mu \mathrm{m}$ & $8.6 \pm 1.2 \mu \mathrm{m}$ & $8.6 \pm 1.2 \mu \mathrm{m}$ \\
\hline Parabasal & $31 \pm 6 \mu \mathrm{m}$ & $31 \pm 6 \mu \mathrm{m}$ & $8.3 \pm 1.0 \mu \mathrm{m}$ & $8.3 \pm 1.0 \mu \mathrm{m}$ \\
\hline Basal & $16 \pm 3.2 \mu \mathrm{m}$ & $16 \pm 3.2 \mu \mathrm{m}$ & $9.0 \pm 1.8 \mu \mathrm{m}$ & $9.0 \pm 1.8 \mu \mathrm{m}$ \\
\hline CIN1 (superficial) & $46 \pm 10 \mu \mathrm{m}$ & $46 \pm 10 \mu \mathrm{m}$ & $8.0 \pm 2.2 \mu \mathrm{m}$ & $8.0 \pm 2.2 \mu \mathrm{m}$ \\
\hline CIN2 (superficial) & $38 \pm 7.1 \mu \mathrm{m}$ & $38 \pm 7.1 \mu \mathrm{m}$ & $8.4 \pm 2.2 \mu \mathrm{m}$ & $8.4 \pm 2.2 \mu \mathrm{m}$ \\
\hline CIN3 (superficial) & $20 \pm 5.5 \mu \mathrm{m}$ & $20 \pm 5.5 \mu \mathrm{m}$ & $13 \pm 2.2 \mu \mathrm{m}$ & $13 \pm 2.2 \mu \mathrm{m}$ \\
\hline
\end{tabular}

\section{Numerical model of the cervical cells}

The extracted contours of the cells are exported in a vector graphics form to COMSOL 5.5 multiphysics software where, these cells are placed in a computational domain of width $500 \mu m \times 500 \mu m$. Copper electrodes are placed on the top and bottom of the computational domain. The parameters used are provided in the supplementary file S1.docx.

The cell membrane is made up of a bi-lipid layer, which when exposed to an electric field causes the dipoles to get pushed away - leading to an "opening" - pore. The pores formed are generally of radii $r_{p}=0.8 \mathrm{~nm}^{5}$ and their creation causes an increase in the conductivity of the cell membrane. These pores formed are represented by a statistical Smoluchowski equation given by:

$$
\frac{\partial N}{\partial t}=\alpha e^{\left.\left(\frac{t m v}{V_{e p}}\right)^{2}\right)}\left[1-\frac{N}{N_{0}} e^{\left(-q\left(\frac{t m v}{V_{e p}}\right)^{2}\right)}\right]
$$

where $N_{0}$ is the initial pore density on the cell membrane $\left(3.3 \times 10^{6} \mathrm{~m}^{-2}\right)$. The $N$ represents temporal pore density, $t m v$ is the trans-membrane potential developed at that instant of time. The $V_{e p}$ represents the characteristic electroporation voltage with $\alpha$ and $q$ representing electroporation constant. The conductivity of the cell membrane increases due to the formation of pores and it represented by an average value:

$$
\sigma(x, y, t)=\sigma_{0}+\pi \sigma_{p} r_{p} N(x, y, t) K
$$

where $\sigma_{0}$ is the static conductivity of the lipid bi-layer. The factor $K$ is calculated as given by the following equation.

$$
K=\frac{e^{v_{m}}-1}{\frac{w_{0} e^{w}-\eta \eta v_{m}}{w_{0}-\eta v_{m}}-\frac{w_{0} e^{w_{0}}+\eta v_{m}}{w_{0}+\eta v_{m}}}
$$

where $w_{0}$ is the energy barrier, $\eta$ is the relative entrance length and $v_{m}=\frac{q_{e}}{K T} V_{m}$ is the compensated transmembrane voltage. By solving the Laplace equation the electric potential is calculated in the simulation domain.

$$
\begin{aligned}
& \nabla \cdot \nabla\left(\sigma+\epsilon_{0} \frac{\partial \phi}{\partial t}\right)-\frac{\partial \nabla \cdot P}{\partial t}=0 \\
& E=-\nabla \phi
\end{aligned}
$$

where $\phi$ represents potential developed in the computational domain. The equation 8-12 and 19 are used in the calculation of developed potential. The Trans-membrane potential is calculated using equation 13.

$$
T M V=\phi_{\text {outer }}(x, y, t)-\phi_{\text {inner }}(x, y, t)
$$

A thin layer is used as boundary impedance for easy computation applied on the plasma membrane given by equation 14 available in COMSOL 5.5a electric current module. The membrane thickness is taken to be $5 \mathrm{~nm}$.

$$
n . J=\frac{\sigma_{m}}{d_{m}}\left(V-V_{r e f}\right)+\frac{\epsilon_{m}}{d_{m}}\left(\frac{\partial V}{\partial t}-\frac{\partial V_{r e f}}{\partial t}\right)
$$


The temporal development of pore radius is given by the equations 15-16.

$$
\frac{d r_{j}}{d t}=\frac{D}{K T}\left(\frac{V_{m}^{2} F_{\max }}{1+r_{h} /\left(r+r_{t}\right)}+4 \beta\left(\frac{r *}{r}\right)^{4} \frac{1}{r}-2 \pi \gamma+2 \pi N \delta_{e f f}\right)
$$

where $V_{m}$ is the transmembrane potential developed during electroporation. The number of hydrophilic pores developed $\left(r>r^{*}\right)$ are from $\mathrm{j}=1,2,3 \ldots k_{p}, \mathrm{~K}$ represents Boltzmann constant, $\delta_{\text {eff }}$ is calculated as:

$$
\delta_{e f f}=2 \delta^{\prime}-\frac{\left(2 \delta^{\prime}-\delta_{0}\right)}{\left(1-\frac{A_{p}}{A}\right)^{2}}
$$

where $\mathrm{A}$ is the area of the plasma membrane and total perforation area is given by $A_{p}$ which is calculated as: ${ }^{5}$

$$
A_{p}=\oiint_{S} N(t) \pi r_{j}^{2} d S
$$

where $\mathrm{S}$ is the plasma membrane surface. The Multi-relaxation Debye-based relationship is used to model the cervical cell dielectric properties. The cytoplasm and nucleus of the cervical cells in this study use a dispersive medium whose dielectric properties have been modeled using a second-order equation ${ }^{6}$.

$$
\begin{aligned}
& \epsilon(\omega)=\epsilon_{\infty}+\frac{\Delta \epsilon_{s}+j \omega\left(\tau_{1} \Delta \epsilon_{2}+\tau_{2} \Delta \epsilon_{1}\right)}{1-\omega^{2} \tau_{p}+j \omega \tau_{s}} \\
& \Delta \epsilon_{s}=\Delta \epsilon_{1}+\Delta \epsilon_{2}, \tau_{p}=\tau_{1} \tau_{2}, \tau_{s}=\tau_{1}+\tau_{2}
\end{aligned}
$$

where $\epsilon_{\infty}$ denotes the high frequency permittivity, $\tau_{1} \& \tau_{2}$ are the relaxation times and $\Delta \epsilon_{1}, \Delta \epsilon_{2}$ are the relaxation amplitudes. The polarization vector $P$ can be expressed in the second-order differential equation which is known as Debye Dispersion model. In this equation, the polarization $P$ is expressed as a time-varying model of a homogeneous medium in terms of a time-varying electric field given by:

$$
\begin{aligned}
& \tau_{p} \frac{d^{2} P}{d t^{2}}+\tau_{s} \frac{d P}{d t}+P=k_{2} \frac{d^{2} E}{d t^{2}}+k_{1} \frac{d E}{d t}+k_{0} E \\
& k_{0}=\Delta \epsilon_{s}+\epsilon_{\infty}-\epsilon_{0}, k_{1}=\tau_{1}\left(k_{0}-\Delta \epsilon_{1}\right)+\tau_{2}\left(k_{0}-\Delta \epsilon_{2}\right), k_{2}=\tau_{p}\left(k_{0}-\Delta \epsilon_{2}\right)
\end{aligned}
$$

\section{Temperature change during electroporation}

The changes in the temperature during electroporation is measured using the Bio-Penes equation ${ }^{25}$ which is defined as:

$$
\nabla \cdot(k \nabla T)+\sigma|\nabla \psi|^{2}+q^{\prime \prime \prime}-W_{b} c_{b} T=\rho c_{p} \frac{\partial T}{\partial t}
$$

where $\mathrm{T}$ is the temperature initially taken as $310.15 \mathrm{~K}, W_{b} c_{b} T$ is heat generated in bloodstream, $q^{\prime \prime \prime}$ is the heat generated in the metabolism, $\mathrm{K}$ is the thermal conductivity , $c_{p}$ is the heat capacity, $\psi$ is the potential developed and $\sigma$ is the conductivity. This equation has been used to observe the temperature developed in the cells during electroporation to analyze whether the thermal stresses developed exceed the safety threshold or not.

\section{Mathematical modeling of drug and dye uptake into cervical cells during RE}

The cell membrane separates the extracellular and intra-cellular fluids and with the help of electrically mediated poration, the drugs \& dyes can be transported. The concentration of drug or dye throughout extracellular space is given by:

$$
\frac{\partial C_{E}}{\partial t}=\left(\vec{\nabla} \cdot\left(D_{e f f} \vec{\nabla} C_{E}\right)-\left(\frac{1-\epsilon}{\epsilon}\right)\left(\frac{\pi R_{p}^{2} N_{p}}{V_{o}}\right) P \cdot\left[\exp (-t / \tau) \cdot S F \cdot\left(C_{E}-C_{R E}\right)+(1-S F) \cdot\left(C_{E}-C_{I R E}\right)\right]\right.
$$


where $C_{E}$ represents the concentration of dye or drug in the extracellular fluid which is taken as $0.01 \mathrm{~mole} / \mathrm{m}^{3}$. The $D_{\text {eff }}=10^{-4} \mathrm{~mm}^{2} \mathrm{sec}^{-1}$ is the effective diffusion of the material in the extracellular fluid is proposed. Since, RE numerical study is observed the Survival Factor (SF) of the cell during electroporation is kept 1 the IRE part is cancelled out. The porosity of membrane $\epsilon$ is 0.18 and resealing time constant of pores $\tau=100 \mathrm{~s}$. The pore radius $R_{P}=0.8 \mathrm{~nm}$ and $\mathrm{P}$ is the permeability of drug and dye kept as $5 \times 10^{-4} \mathrm{~mm} / \mathrm{sec}$. $V_{o}$ represents the volume of the cube in which the cell is kept which kept.

$$
\frac{\partial C_{R E}}{\partial t}=\left(\frac{\pi R_{p}^{2} N_{p}}{V_{o}}\right) P .\left[\exp (-t / \tau) \cdot S F \cdot\left(C_{E}-C_{R E}\right)\right]
$$

where $C_{R E}$ represents the concentration of dye or drug in the intracellular region of the cell. The initial conditions of $C_{E}=0.01 \mathrm{~mole} / \mathrm{m}^{3}$ and $C_{R E}=0$. The no flux boundary conditions are given as $\frac{\partial C_{R E}}{\partial r}=0$ and $\frac{\partial C_{E}}{\partial r}=0^{26}$.

\section{Conclusion}

This work is an aggolomerative approach wherein the two-dimensional contours of the overlapping and freestanding cervical cells were successfully extracted with the help of an algorithm developed using the level set method.This was followed by a mathematical model to study RE - which was successfully validated against experimental data. The simulation study is done for cellular clumps of different orientations with a different number of cells and for isolated cells combined with overlapping cells. For every orientation, we observe that threshold has been achieved for reversible electroporation proving the validity of the proposed model. The temperature analysis is done for all the models to highlight that the thermal envelop is not breached - which indicates significantly low ablation likelihood. We subsequently apply high-frequency pulse to the model of normal and cancerous cells. The cervical cells of various types including those at varying stages of neoplasia, have been used. The simulations show that the normal cells exhibit a delayed and attenuated responses compared to the rest. The use of drug and dye delivery diffusion equation has also been used to show the molar uptake by the cells is modulated. The pore evaluation of normal and cancerous cells has been analyzed, showing faster evolution in cancerous cells. These model results could be further used for microdosimetry analysis that employs a simple image processing algorithm contour extraction. The dyes uptake change could be further be used to analyze morphology change in the cervical cells - potentially indicating onset and progression of Intraepithilial neoplasia. Electrically mediate drug uptake could also be explored for electro-chemotherapy. Natural extension of this work points to model-driven approach microdosimetry study for 3D cervical normal and cancerous cells by image domain volumetric extraction of overlapping isolated cells. Towards this end, a low-cost simple nano-pulse generator has already been proposed ${ }^{18}$.

\section{References}

1. T. Kotnik, L. Rems, M. Tarek, and D. Miklav`ci`c, "Membrane electroporation and electropermeabilization: Mechanisms and models." Ann. Rev. Biophys., vol. 6, no. 48, pp. 63-91, 2019.

2. Miklavcic D, Puc M (2006) Electroporation, Wiley Encyclopedia of Biomedical Engineering. John Wiley, Newyork

3. De Angelis Annalisa, Denzi Agnese, Merla Caterina, Andre Frank M., Mir Lluis M., Apollonio Francesca, Liberti Micaela,Confocal Microscopy Improves 3D Microdosimetry Applied to Nanoporation Experiments Targeting Endoplasmic Reticulum.Frontiers in Bioengineering and Biotechnology, doi:10.3389/fbioe.2020.552261 ,ISBN:2296-4185.

4. C. Merla, A. Paffi, F. Apollonio, P. Leveque, G. d'Inzeo and M. Liberti, "Microdosimetry for Nanosecond Pulsed Electric Field Applications: A Parametric Study for a Single Cell," in IEEE Transactions on Biomedical Engineering, vol. 58, no. 5, pp. 1294-1302, May 2011, doi: 10.1109/TBME.2010.2104150.

5. Krassowska W, Filev PD. Modeling electroporation in a single cell. Biophys J. 2007 Jan 15;92(2):404-17. doi: 10.1529/biophysj.106.094235. Epub 2006 Oct 20. PMID: 17056739; PMCID: PMC1751390.

6. E. Salimi, D. J. Thomson and G. E. Bridges, "Membrane dielectric dispersion in nanosecond pulsed electroporation of biological cells," in IEEE Transactions on Dielectrics and Electrical Insulation, vol. 20, no. 4, pp. 1256-1265, August 2013, doi: 10.1109/TDEI.2013.6571442.

7. Pucihar G, Miklavcic D, Kotnik T. A time-dependent numerical model of transmembrane voltage inducement and electroporation of irregularly shaped cells. IEEE Trans Biomed Eng. 2009 May;56(5):1491-501. doi: 10.1109/TBME.2009.2014244. Epub 2009 Feb 6. PMID: 19203876. 
8. L. Mescia, M. A. Chiapperino, P. Bia, J. Gielis and D. Caratelli, "Modeling of Electroporation Induced by Pulsed Electric Fields in Irregularly Shaped Cells," in IEEE Transactions on Biomedical Engineering, vol. 65, no. 2, pp. 414-423, Feb. 2018, doi: 10.1109/TBME.2017.2771943.

9. Chiapperino MA, Bia P, Caratelli D, Gielis J, Mescia L, Dermol-Černe J, Miklavčič D. Nonlinear Dispersive Model of Electroporation for Irregular Nucleated Cells. Bioelectromagnetics. 2019 Jul;40(5):331-342. doi: 10.1002/bem.22197. Epub 2019 Jun 9. PMID: 31179573.

10. Denzi A, Camera F, Merla C, Benassi B, Consales C, Paffi A, Apollonio F, Liberti M. A Microdosimetric Study of Electropulsation on Multiple Realistically Shaped Cells: Effect of Neighbours. J Membr Biol. 2016 Oct;249(5):691-701. doi: 10.1007/s00232-016-9912-3. Epub 2016 Jun 18. PMID: 27318672.

11. M. A. Chiapperino et al., "Experimental and Numerical Study of Electroporation Induced by Long Monopolar and Short Bipolar Pulses on Realistic 3D Irregularly Shaped Cells," in IEEE Transactions on Biomedical Engineering, vol. 67, no. 10, pp. 2781-2788, Oct. 2020, doi: 10.1109/TBME.2020.2971138.

12. Ferlay J, Soerjomataram I, Dikshit R, Eser S, Mathers C, Rebelo M, Parkin DM, Forman D, Bray F. Cancer incidence and mortality worldwide: sources, methods and major patterns in GLOBOCAN 2012. Int J Cancer. 2015 Mar 1;136(5):E359-86. doi: 10.1002/ijc.29210. Epub 2014 Oct 9. PMID: 25220842.

13. Das L, Das S, Chatterjee J. Electrical Bioimpedance Analysis: A New Method in Cervical Cancer Screening. J Med Eng. 2015;2015:636075. doi: 10.1155/2015/636075. Epub 2015 Feb 2. PMID: 27006939; PMCID: PMC4782745.

14. D.C. Walker; B.H. Brown; D.R. Hose; R.H. Smallwood,"Modelling the electrical impedivity of normal and premalignant cervical tissue", Electronics Letters ( Volume: 36, Issue: 19, 14 Sep 2000)

15. D C Walker 1, B H Brown, R H Smallwood, D R Hose, D M Jones,"Modelled current distribution in cervical squamous tissue",Physiol Meas . 2002 Feb;23(1):159-68. doi: 10.1088/0967-3334/23/1/315.

16. Yongji Yang,Michael A. J. Moser,Edwin Zhang,Wenjun Zhang ,Bing Zhang,"Development of a statistical model for cervical cancer cell death with irreversible electroporation in vitro",Published: April 25, 2018https://doi.org/10.1371/journal.pone.0195561

17. K. Mitsutake, A. Satoh, S. Mine, K. Abe, S. Katsuki and H. Akiyama, "Effect of pulsing sequence of nanosecond pulsed electric fields on viability of HeLa S3 cells," in IEEE Transactions on Dielectrics and Electrical Insulation, vol. 19, no. 1, pp. 337-342, February 2012, doi: 10.1109/TDEI.2012.6148536.

18. Kumar, Mayank and Mishra, Ashutosh,"Reversible electroporation study of realistic normal and cancerous cervical cells model using avalanche transistor-based nano pulse generator," IOP, Biomedical Physics Engineering Express ;http://iopscience.iop.org/article/10.1088/2057-1976/ac240b.

19. https://cs.adelaide.edu.au/ zhi/project/TIP CellSegJointL $S_{2} 014 /$ Dataset.zip

20. D. Comaniciu and P. Meer, "Mean shift: A robust approach toward feature space analysis," IEEE Trans. Pattern Anal. Mach. Intell., vol. 24, no. 5, pp. 603-619, May 2002.

21. J. Matas, O. Chum, M. Urban, and T. Pajdla, "Robust wide baseline stereo from maximally stable extremal regions," in Proc. BMVC, Cardiff, U.K., 2002, pp. 384-396.

22. C. Li, C. Xu, C. Gui and M. D. Fox, "Distance Regularized Level Set Evolution and Its Application to Image Segmentation," in IEEE Transactions on Image Processing, vol. 19, no. 12, pp. 3243-3254, Dec. 2010, doi: 10.1109/TIP.2010.2069690.

23. Z. Lu, G. Carneiro and A. P. Bradley, "An Improved Joint Optimization of Multiple Level Set Functions for the Segmentation of Overlapping Cervical Cells," in IEEE Transactions on Image Processing, vol. 24, no. 4, pp. 1261-1272, April 2015, doi: 10.1109/TIP.2015.2389619.

24. Gorazd Pucihar, T. Kotnik, D. Miklavcic,"An experimental and numerical study of the induced transmembrane voltage and electroporation on clusters of irregularly shaped cells," in 11th Mediterranean Conference on Medical and Biomedical Engineering and Computing 2007,DOI: 10.1007/978.3.540.73044.6.166.

25. Khorasani,"The Effect of Conductivity Changes on Temperature Rise During Irreversible Electroporation", number:3, Frontiers in Biomedical Technologies, September 2020, pages:178-185,doi:10.18502/fbt.v7i3.4620.

26. Nilay Mondal, Koyel Chakravarty, and D. C. Dalal1,"Mathematical modeling of drug delivery in tissue cells using electroporation,"AIP Conference Proceedings 1975, 030017 (2018); https://doi.org/10.1063/1.5042187 


\section{Supplementary Files}

This is a list of supplementary files associated with this preprint. Click to download.

- S1.docx 